\title{
Sepsis Screening Tools
}

\author{
Amanda Venable MSN, RN, CCRN
}

\section{CASE}

Mr. $\mathrm{H}$ is a 67-year-old man status post hemicolectomy four days ago. He was transferred from the ICU to a medical-surgical floor at 1700 last night. Overnight the nurse called the house officer regarding urine output less than $0.5 \mathrm{ml} / \mathrm{kg} / \mathrm{hr}$ and tachycardia of 105 beats per minute. The house officer ordered a one liter NS bolus. This morning the patient is exhibiting signs and symptoms of severe sepsis, including temperature $103.5^{\circ} \mathrm{F}$, HR 117 beats per minute, mean arterial pressure $58 \mathrm{mmHg}$, decreased level of consciousness, and decreased urine output. His WBC is $21,000 / \mu \mathrm{L}$. The patient is now critically ill and is being transferred to a critical care unit. Could there have been a better way to identify the problem the night before and intervene before this change in status became severe?

\section{Discussion}

Scenarios like the one above occur commonly in hospitals. A study of septic patients in a surgical intensive care unit (SICU) showed that $47 \%$ of the patients admitted with sepsis, severe sepsis, or septic shock came from a surgical floor ${ }^{1}$. Patients who develop sepsis as inpatients present different challenges from patients who present to the hospital with sepsis.

According to the Institute for Healthcare Improvement $(\mathrm{IHI})$, the incidence of sepsis has increased to 750,000 new cases per year with at least 210,000 fatalities ${ }^{2}$. Seventeen percent of hospitalizations with

\section{Corresponding author: Amanda Venable MSN,} RN, CCRN

Contact Information: Amanda.venable@umchealth system.com

DOI: $10.12746 /$ swrccc2013.0103.027 the diagnosis of septicemia or sepsis result in death compared with only $2 \%$ of other types of hospital admissions ${ }^{2}$. Besides having a high mortality rate, sepsis can cause long periods of debilitation. Thirtysix percent of patients hospitalized with sepsis are transferred to other facilities, such as long term care, compared to $14 \%$ of other types of inpatients. Implementation of Early Goal Directed Therapy (EGDT) improves sepsis survival ${ }^{3}$. However, early recognition of sepsis in the inpatient setting can be a challenge.

Early recognition of sepsis is imperative in improving mortality rates. A 2001 study showed improvement in mortality rates only if EGDT is initiated within six hours ${ }^{4}$. The challenge is the complexity of sepsis which prevents early recognition from occurring consistently, particularly on medical-surgical units. However, clinical knowledge alone does not guarantee sepsis will be recognized. The clinician must have time to review patient data and determine whether the patient has signs of sepsis. This is challenging in today's fast-paced clinical environment. The complexities of sepsis recognition can be overcome by implementing a systematic recognition program for sepsis. Screening tools are widely available and are effective in the recognition of sepsis ${ }^{4-6}$.

It is important for each healthcare facility to choose a method for screening which is congruent with the workflow of the facility. A few of the considerations include:

1. Who should do the screening?

2. How often should the screening be done?

3. Should the screening be done on paper or electronically?

Although physicians and other healthcare providers have the ultimate responsibility in determining if a patient is septic, their contact with the patient is 
limited compared to the contact the nursing staff has with the patient. The requirement for early recognition makes it necessary for the nursing staff to be able to recognize potential sepsis in the patients and report findings to healthcare providers. Many institutions utilize the primary nurse for completing screening tools while others use charge nurses or rapid response teams ${ }^{7,8}$. The frequency of screening is also a difficult question because some of the SIRS criteria, such as lab values, would have data points only once a day, while others, such vital signs, have more continuous assessments. Studies have examined screening tools used at a wide range of frequencies. Some screening tools are completed only on admission and with any sign of patient deterioration; others are done every time a new set of vital signs is entered into the medical record. Hospitals still using paper charting will implement a paper sepsis screening tool. Hospitals with electronic medical records may consider partially automating the screening tool using data already entered by nursing and ancillary staff. A multidisciplinary team should be formed in each institution to determine the best method for sepsis screening for the facility ${ }^{7}$.

The University Medical Center Health System Critical Care Collaborative is a multidisciplinary team formed to improve the quality of critical care delivered in the facility. The Collaborative determined the need for a sepsis screening tool and developed one individualized for the facility based mostly on the screening tools available on the IHI website (Figure 1). The screening tool was completed by the primary care nurse on admission to the intensive care unit to determine its potential utility. After a one month trial it was determined the tool was adequate for identifying sepsis. However, use of the tool was not consistent because the tool was on paper and our hospital utilizes an electronic medical record. The Critical Care Collaborative worked with information technology professionals to develop an electronic sepsis screening tool. This tool works by "firing a rule" every time a nurse completes a head to toe assessment on the patient. The rule prompts the computer system to look for criteria identifying sepsis as the paper sepsis screening tool does. If the patient is identified as potentially having sepsis according to the computer, a task is "fired" for the primary nurse to complete a sepsis screening tool. Figure 2 is a screen shot of the electronic sepsis screening tool. Only time will tell if the electronic sepsis screening tool will be effective for the UMC Health System. Adjustments may be needed to create the most efficient and accurate sepsis screening tool. Continuous evaluation of the tool's effectiveness by the multidisciplinary Critical Care Collaborative will insure a method for improving the recognition of sepsis in our healthcare facility.

\section{KEY POINTS}

1. Sepsis has a high morbidity and mortality.

2. Inpatients who develop sepsis may have delays in evaluation, testing, and treatment.

3. Sepsis screening tools based on SIRS criteria can provide a rapid method to help identify sepsis.

4. These tools are potentially useful for all health care providers but need to be used consistently.

KEY WORDS- sepsis, screening, electronic record, surviving sepsis guidelines 
Figure 1. Paper Sepsis Screening Tool

\section{University Medical Center Adult ICU \\ Sepsis Screening Tool}

Step 1:

Is the patient already being treated for sepsis?

Y Yes _ No

If answer is yes, STOP.

If answer is no, CONTINUE to step 2

Step 2: (Two or more of the following)

A) Sepsis Criteria

Temp $>100.9$ or $<96.8$ (in the last 24 hours)

$\mathrm{HR}>90 \quad$ (in the last 24 hours)

Respiratory Rate $>20$ or $\mathrm{PaCO} 2<32$ (in the last 24 hours)

WBC $>12000$, < 4000 , or $>10 \%$ Bands

If less than two items checked, STOP.

\section{Step 3: Infection (Suspected or Confirmed)}

Does this patient have a suspected or confirmed source of infection?

Yes

Place Patient Label Here

(Such as: Pneumonia, Invasive Catheter, UTI, Decubitis Ulcer, Acute Abdomen, Colitis, Meningitis, Pancreatitis, Cellulitis, Bone/Joint, or Wound)

If answer is NO, STOP.

If answer is YES, continue to step 4 and contact physician if necessary. The patient may have SEPSIS.

\section{Step 4: Organ Dysfunction}

Acutely altered mental status

SBP $<90$ or MAP $<65$

$\mathrm{SPO} 2<90 \%$

Creatinine $>2 \mathrm{mg} / \mathrm{dl}$ or urine output $<0.5 \mathrm{mg} / \mathrm{kg} / \mathrm{hr}$

Platelet count $<100,000$

Bilirubin $>2 \mathrm{mg} / \mathrm{dl}$, AST $>90$, ALT $>90$

Lactate $>2 \mathrm{mmol} / \mathrm{L}$

If one or more items are checked the patient may have SEVERE SEPSIS.

Step 5. If patient screens positive for SEPSIS or SEVERE SEPSIS, CALL PHYSICIAN NOW (if not already aware).

Early Goal Directed Therapy for Adult Sepsis orders were implemented

WHY

$$
\text { Early Goal Directed Therapy for Adult Sepsis orders were NOT implemented }
$$

Date

Time

Not Part of the Medical Record 
Figure 2. Electronic Sepsis Screening Tool Sepsis Screening Tool (Complete form)

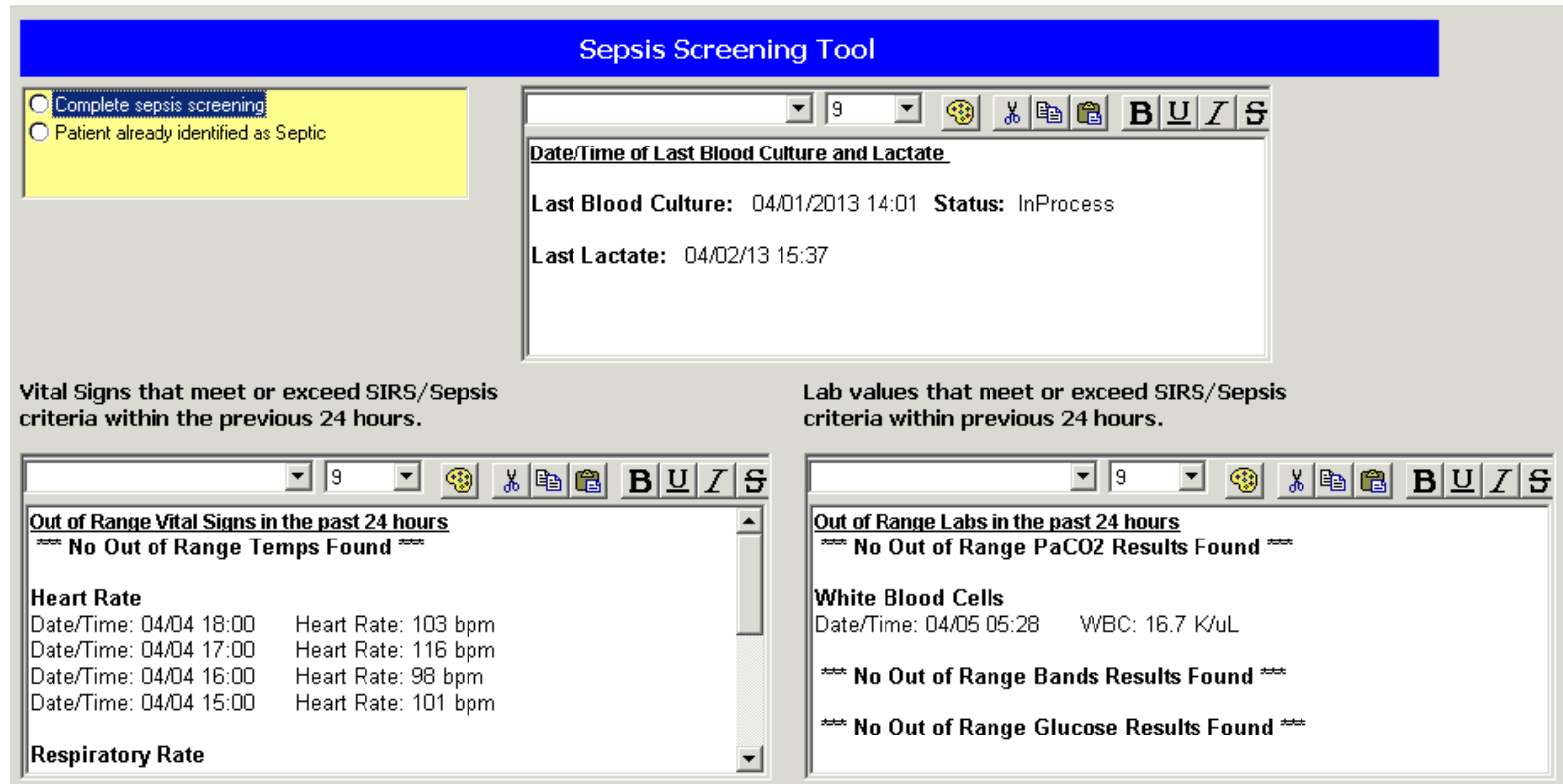

Does the patient meet any of the following SIRS criteria?
Does the patient have a suspected or confirmed infection?

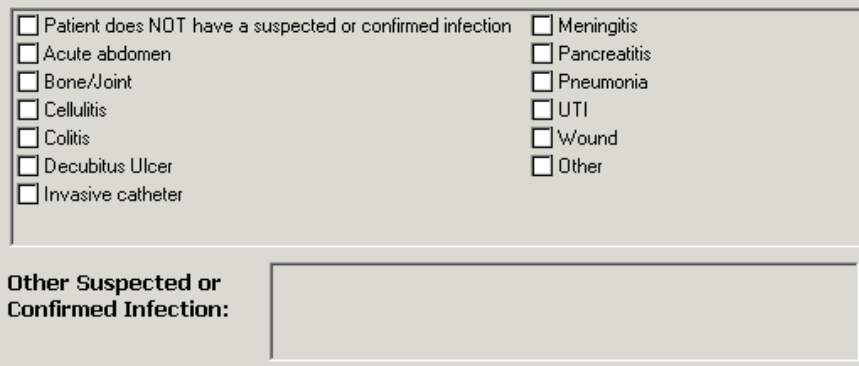

Lab values that meet or exceed Organ Dysfunction criteria within the previous 24 hours.

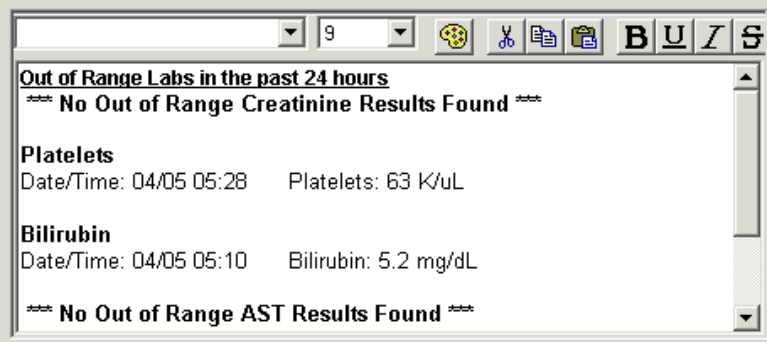

criteria within the previous 24 hours.

\begin{tabular}{|c|c|c|c|c|c|c|}
\hline & $=9$ & ㄱ웡 & 另 & $\mathbf{B}|\underline{\mathbf{U}}|$ & $Z$ & 5 \\
\hline \multicolumn{7}{|c|}{ Out of Range Vital Signs in the past 24 hours } \\
\hline \multicolumn{7}{|c|}{ No Out of Range SBPs Found } \\
\hline \multicolumn{7}{|l|}{ Mean Arterial Pressure } \\
\hline Date/Time: 04/04 15:00 & MAP: & $\mathrm{mmHg}$ & & & & \\
\hline$\Rightarrow$ No Out of Range $0 x$ & ygen $s$ & Found & & & & \\
\hline
\end{tabular}

Does the patient meet any of the following Organ Dysfunction signs/symptoms criteria?

$\square$ Patient does NOT meet any of the listed criteria

$\square$ Acutely altered mental status

$\square$ SpO2 less than $90 \%$

$\square$ Platelet count less than 100,000

Lactate greater than $2 \mathrm{mmol} / \mathrm{L}$

$\square$ SBP less than 90 or MAP less than 65

$\square$ Creatinine $>2 \mathrm{mg} / \mathrm{dL}$ or urine output $<0.5 \mathrm{mg} / \mathrm{kg} / \mathrm{hr}$

$\square$ Bilirubin $>2 \mathrm{mg} / \mathrm{dL}$, AST $>90$, ALT $>90$
If the patient meets any of the Organ Dysfunction signs/symptoms criteria, then please notify the primary team. A task will follow where the notification can be documented.

Rapid Response Team Notified

OYes

O No

N/A 


\section{Sepsis Screening Tool (In Sections)}

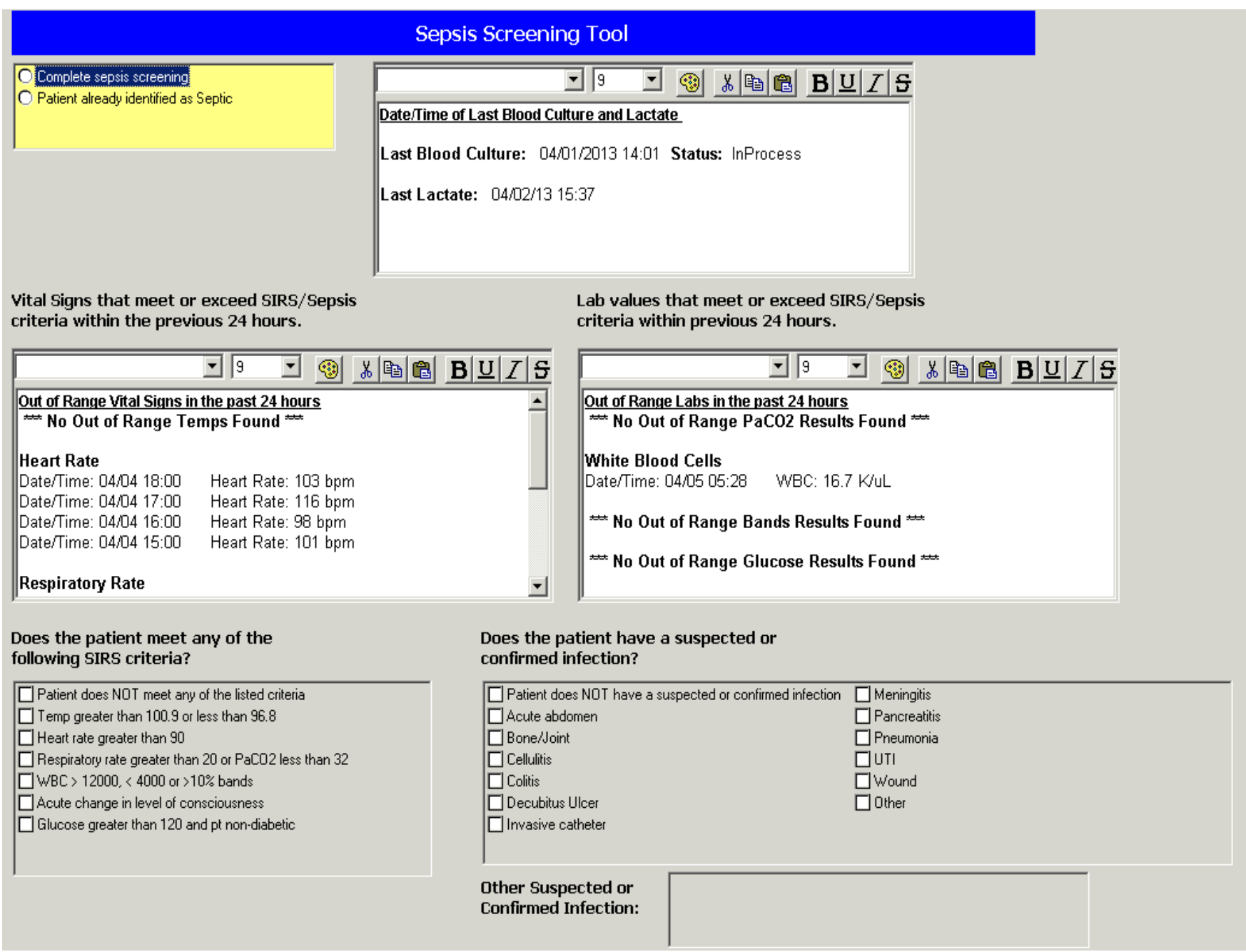

Vital Signs that meet or exceed Organ Dysfunction criteria within the previous 24 hours.

\begin{tabular}{|l}
\hline Out of Range Vital Signs in the past 24 hours \\
No Out of Range SBPs Found \\
Mean Arterial Pressure \\
Date/Time: $04 / 04$ 15:00 MAP: $64 \mathrm{mmHg}$ \\
No Out of Range Oxygen Sats Found
\end{tabular}

Lab values that meet or exceed Organ Dysfunction criteria within the previous 24 hours.

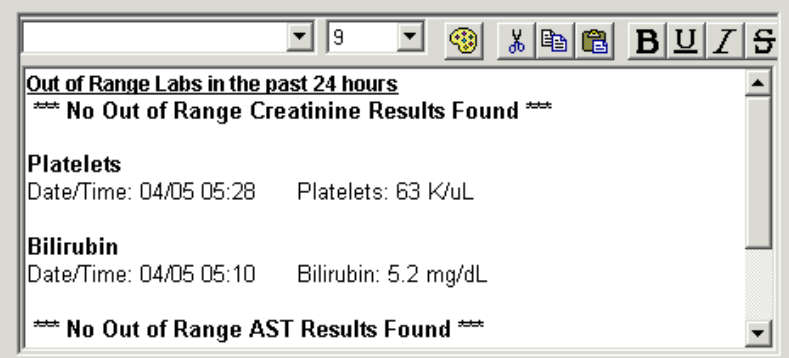

Does the patient meet any of the following Organ Dysfunction signs/symptoms criteria?

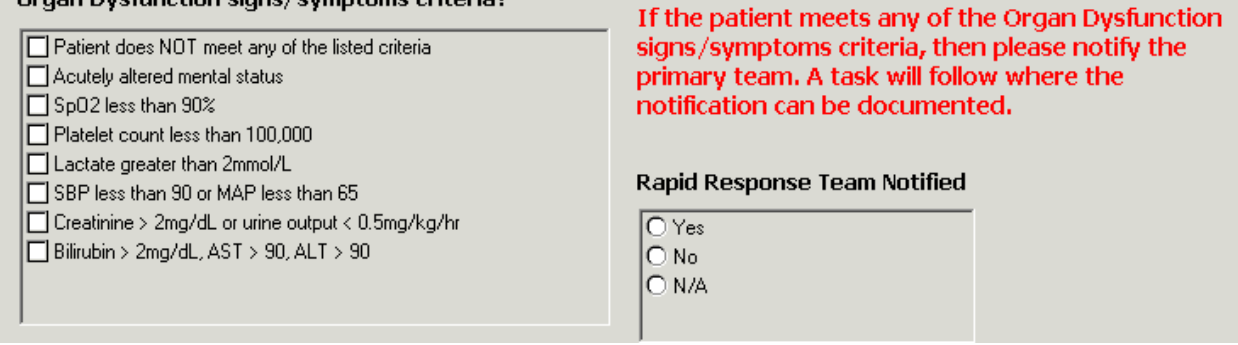



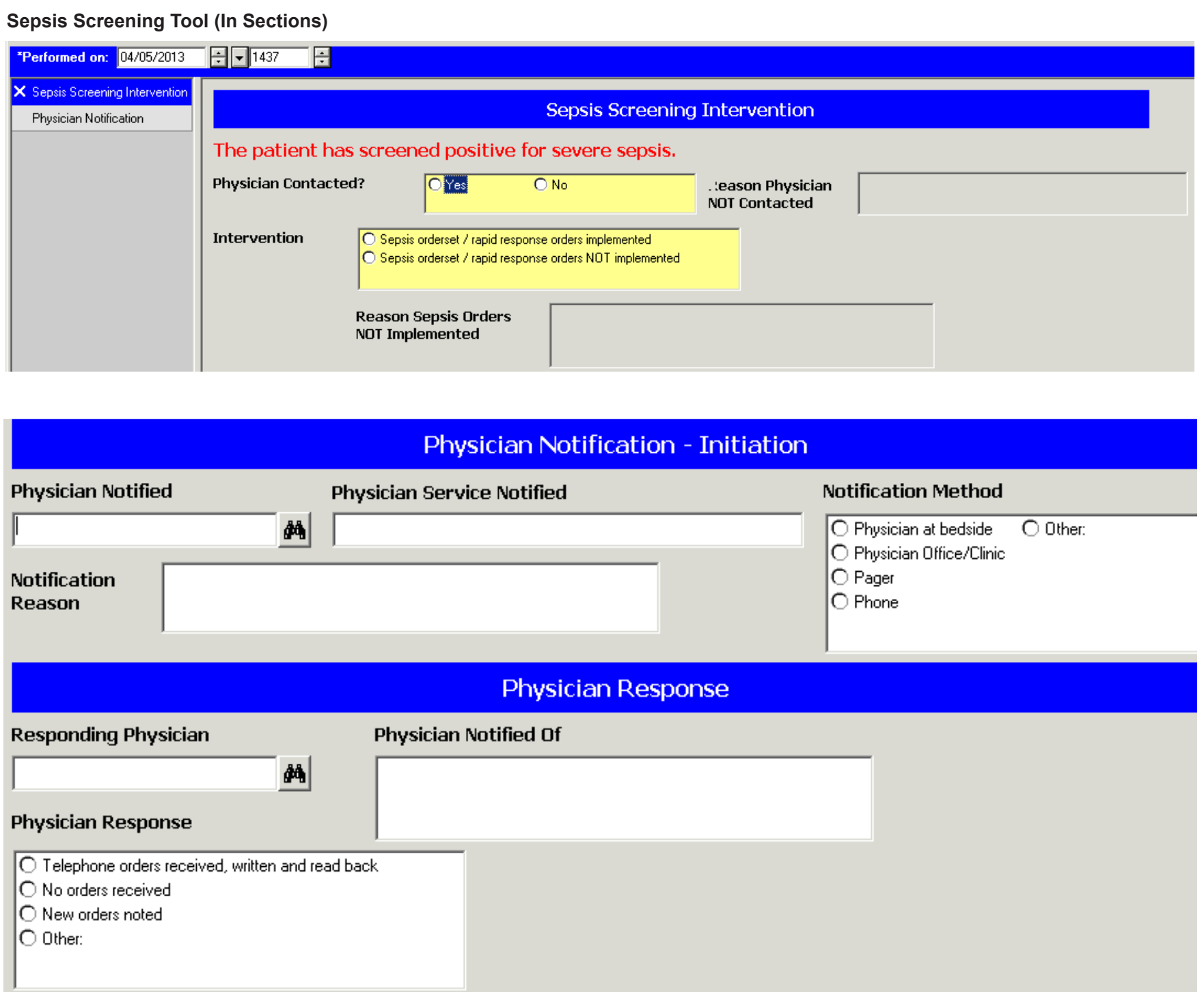

Author Affiliation: Amanda Venable is the Nurse Director for the SICU and BICU at University Medical Center in Lubbock, TX.

Received: $1 / 23 / 2013$

Accepted: $6 / 3 / 2013$

Reviewers: R Alalawi MD, K Nugent MD

Published electronically: 7/15/2013

Conflict of Interest Disclosures: None

\section{REFERENCES}

1. National Center for Health Statistics (2010 preliminary data); available at www.cdc.gov.

2. Pauwels Ra, Bruist AS, Calverley CR, et al. Global strategy for the diagnosis, management, and prevention of chronic obstructive pulmonary disease, NHLBI / WHO workshop summary. Am J Respir Crit Care Med 2001; 163:1256-1276.

3. Curtis JR. palliative and end-of-life care for patients with severe COPD. Eur Respir J 2008; 32:796-803.

4. Local Coverage Determination (LCD) for Hospice Cardiopulmonary Conditions (L31540). Federal Register, V. 70, No. 224, dated Tuesday, November 22, 2005, p. 70537. 
5. Gade $G$, Venohr I, Conner D, et al. Impact of an inpatient palliative care team: a randomized controlled trial. J of Palli Med. 2008; 11(2):180-190.

6. Fromme EK, Bascom PB, Smith SW, et al. Survival, mortality, and location of death for patients seen by a hospital-based palliative care team. J of Palli Med. 2006; 9(4):903-911.

7. Morrison RS, Penrod JD, Cassel JB, et al. Cost savings associated with US hospital palliative care consultation programs. Arch Intern Med. 2008; 168(16): 1783-1790. 